\section{Structural basis of protein complex formation and reconfiguration by polyglutamine disease protein Ataxin-1 and Capicua}

\author{
Eunji Kim, ${ }^{1}$ Hsiang-Chih $\mathrm{Lu}^{2,3}$ Huda Y. Zoghbi, ${ }^{2,3,4,5}$ \\ and Ji-Joon Song ${ }^{1,6}$ \\ ${ }^{1}$ Department of Biological Sciences, Graduate School \\ of Nanoscience and Technology (World Class University), \\ KAIST (Korea Advanced Institute of Science and Technology) \\ Institute for the BioCentury, KAIST, Daejeon 305-701, Korea; \\ ${ }^{2}$ Program in Developmental Biology, Baylor College of \\ Medicine, Houston, Houston, Texas 77030, USA; ${ }^{3}$ Jan and Dan \\ Duncan Neurological Research Institute at Texas Children's \\ Hospital, Houston, Texas 77030, USA; ${ }^{4}$ Department of \\ Molecular and Human Genetics, Baylor College of Medicine, \\ Houston, Texas 77030, USA; ${ }^{5}$ Howard Hughes Medical \\ Institute, Houston, Texas 77030, USA
}

Spinocerebellar ataxia type 1 (SCA1) is a dominantly inherited neurodegenerative disease caused by polyglutamine expansion in Ataxin-1 (ATXN1). ATXN1 binds to the transcriptional repressor Capicua (CIC), and the interaction plays a critical role in SCA1 pathogenesis whereby reducing CIC levels rescues SCA1-like phenotypes in a mouse model. The ATXN1/HBP1 (AXH) domain of ATXN1 mediates its homodimerization as well as the interaction with CIC. Here, we present the crystal structure of ATXN1's AXH domain bound to CIC and show that the binding pocket of the $\mathrm{AXH}$ domain to CIC overlaps with the homodimerization pocket of the AXH domain. Thus, the binding to CIC disrupts the homodimerization of ATXN1. Furthermore, the binding of CIC reconfigures the complex to allow another form of dimerization mediated by CIC, showing the intricacy of protein complex formation and reconfiguration by ATXN1 and CIC. Identifying the surfaces mediating the interactions between CIC and ATXN1 reveals a critical role for $\mathrm{CIC}$ in the reconfiguration of the $\mathrm{AXH}$ dimers and might provide insight into ways to target the ATXN1/CIC interactions to modulate SCA1 pathogenesis.

Supplemental material is available for this article.

Received December 11, 2012; revised version accepted February 7, 2013.

Spinocerebellar ataxia type 1 (SCA1) is one of nine polyglutamine (polyQ) expansion neurodegenerative diseases. SCA1 is caused by the polyQ expansion in Ataxin-1 (ATXN1) (Orr et al. 1993). It is believed that the polyQ

[Keywords: complex; neurodegenerative disease; SCA1]

${ }^{6}$ Corresponding author

E-mail songj@kaist.ac.kr

Article is online at http://www.genesdev.org/cgi/doi/10.1101/gad.212068.112. expansion in ATXN1 modulates its normal activity to cause the pathology (Lim et al. 2008) ATXN1 has many binding partners, including Capicua (CIC), a transcriptional repressor that is involved in SCA1 pathogenesis. In fact, reducing CIC levels by $50 \%$ rescues many SCA 1 -like phenotypes in a mouse model of the disease (Fryer et al. 2011). A highly conserved ATXN1/HBP1 (AXH) domain in ATXN1, which is required for SCA1 pathogenesis (Tsuda et al. 2005), interacts with an N-terminal region of CIC (Lam et al. 2006). Furthermore, the AXH domain is required for ATXN1 self-association, which is also shown by the crystal structure of the AXH domain alone (Burright et al. 1997; Chen et al. 2004). Although ATXN1 interacts with several cellular partners and regulates their activities, the biochemical properties of ATXN1 and the molecular mechanism mediating complex formation of ATXN1 with a protein such as CIC are still not well understood. Here, we present the crystal structures of ATXN1's AXH domain bound with CIC peptides. These structures, followed by biochemical and in vivo analysis, reveal that the binding of CIC to ATXN1 dissociates the ATXN1 homodimer and induces a new form of ATXN1 dimer mediated by CICs. These data implicate that ATXN1 undergoes reconfiguration of complex formation upon CIC binding.

\section{Result and Discussion}

\section{Crystal structure of the ATXN1-CIC complex}

To understand the molecular mechanism mediating ATXN1-CIC interaction, we determined the crystal structure of ATXN1's AXH domain bound to a highly conserved N-terminal region of CIC (28-48 amino acids, $\mathrm{CIC}_{21}$ : EPRSVAVFPWHSLVPFLAPSQ) at $2.5 \AA$ resolution using the single-wavelength anomalous dispersion (SAD) method (Supplemental Table 1; Supplemental Fig. S1). The structure of the AXH domain bound with CIC peptide is almost identical with the structure of the AXH domain alone (Chen et al. 2004). The complex structure reveals that $\mathrm{CIC}_{21}$ binds to a highly hydrophobic pocket of the AXH domain (Fig. 1). Highly conserved residues (W37, L40, and V41) of $\mathrm{CIC}_{21}$ form hydrophobic interactions with highly conserved residues (V591, S602, and L686) of the AXH domain (Fig. 1). To confirm that the interaction observed in the crystal structure is biologically relevant, we first mutagenized the conserved residues of $\mathrm{CIC}_{21}$ and examined the binding by maltose-binding protein (MBP) pull-down assay (Fig. 2A, top). Among the conserved residues, mutating the conserved W37 to A (W37A), and L40 to $\mathrm{S}$ (L40S) of $\mathrm{CIC}_{21}$ severely impaired the binding abilities of $\mathrm{CIC}_{21}$ to the $\mathrm{AXH}$ domain, while mutating other conserved residues moderately affected the binding abilities. We next generated several mutations in the hydrophobic pocket of the AXH domain. Among these mutants, combined mutations in the pocket (V591A_S602D, V591A_L686E, or S602D_L686E) abolish the interaction between the $\mathrm{AXH}$ domain and $\mathrm{CIC}_{21}$ (Fig. 2A, bottom).

To determine whether the interaction between the $\mathrm{AXH}$ domain and CIC observed in the crystal structure and whether in vitro pull-down assays reflect in vivo interactions in the context of the full-length proteins, we transfected either Myc-tagged full-length $\mathrm{CIC}\left(\mathrm{CIC}_{\mathrm{f}_{-} \mathrm{WT}}\right)$ 
A

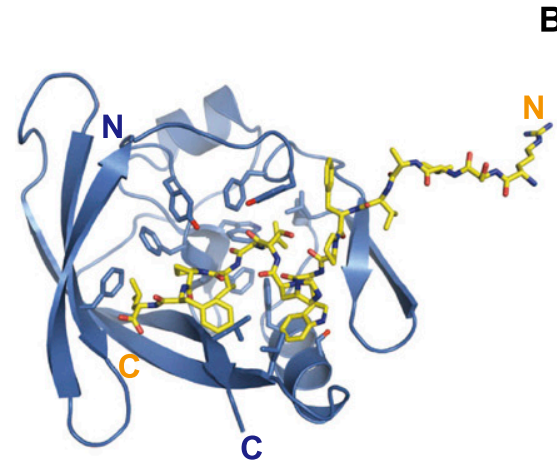

B

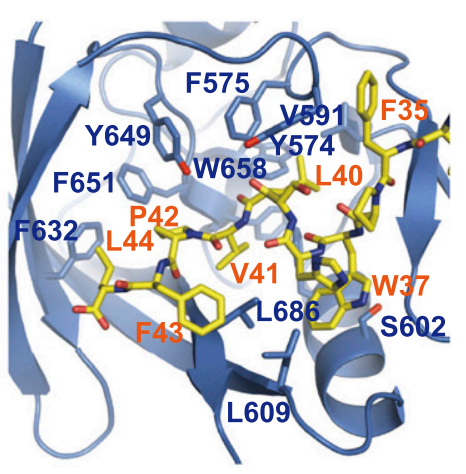

C

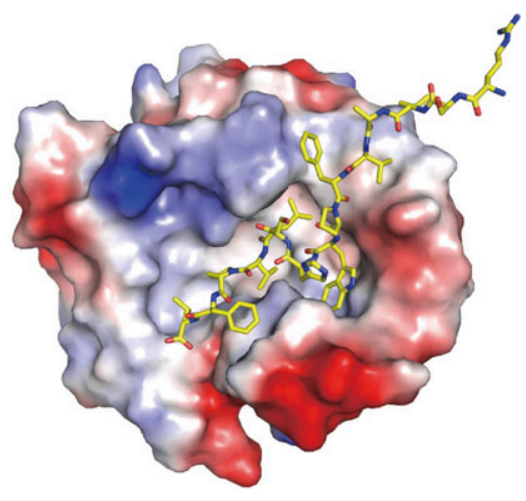

Figure 1. Crystal structure shows that $\mathrm{CIC}_{21}$ binds to a highly hydrophobic pocket of the $\mathrm{AXH}$ domain. $(A)$ Ribbon representation of the $\mathrm{AXH}$ domain (blue) and the bound $\mathrm{CIC}_{21}$ peptide shown in ball-and-stick (yellow). (B) Detailed interaction between the AXH domain and CIC ${ }_{21}$ peptide showing that $\mathrm{CIC}_{21}$ binds to the hydrophobic pocket in the $\mathrm{AXH}$ domain. $(C)$ Electrostatic surface representation of the AXH domain bound with $\mathrm{CIC}_{21}$ (yellow) showing hydrophobic character of the pocket.

or full-length CIC mutants $\left(\mathrm{CIC}_{\mathrm{f}_{-} \text {W37A }}\right.$ and $\left.\mathrm{CIC}_{\mathrm{f}_{\_} \mathrm{L} 40 \mathrm{~S}}\right)$ and asked whether the mutations disrupt the interaction between endogenous ATXN1 and CIC in vivo. Consistent with the structural and in vitro binding data, the conserved hydrophobic residues (W37 and L40) of CIC are critical for ATXN1 binding in vivo (Fig. 2B). Next, we transfected either Flag-tagged full-length wild-type or mutant ATXN1 and examined the binding abilities with endogenous CIC. We found that the AXH mutants (ATXN1 V591A_S602D, $_{\text {, }}$ ATXN1 $1_{\text {V591A_L686E, }}$ and ATXN1 S602D_L686E $_{\text {f }}$ fail to interact with endogenous $\mathrm{CIC}$, but a negative control mutant (R665A) shows binding comparable with that of wild-type ATXN1 (Fig. 2C). These data validate the information deduced from the crystal structure.

\section{Disruption of ATXN1 homodimerization upon CIC binding}

The previous structure of the AXH domain shows that the AXH domain forms a homodimer (Chen et al. 2004). Interestingly, the same pocket on ATXN1 that mediates CIC binding was initially identified to be involved in homodimerization of the AXH domain, suggesting that CIC binding might disrupt AXH domain homodimerization (Supplemental Fig. 2A,B; Chen et al. 2004). Therefore, we investigated whether the CIC binding to the AXH domain disrupts AXH homodimerization by size exclusion chromatography (Supplemental Fig. 3). While the AXH domain alone elutes at fractions corresponding to the AXH dimer, the $\mathrm{AXH}$ domain bound to $\mathrm{CIC}_{21}$ $\left(\mathrm{AXH}_{-} \mathrm{CIC}_{21}\right)$ elutes in a fraction similar to the $\mathrm{N}$-terminal-deleted AXH domain (AXH_ $\Delta N$ ), which is supposed to be a monomer, since the $\bar{N}$ terminus of the $\mathrm{AXH}$ domain is critical for its homodimerization. Combined with the structure of AXH-CIC complex, these data show that the binding of CIC to the hydrophobic pocket of the AXH domain disrupts the homodimerization of ATXN1.

\section{ATXN1-CIC interactions}

To measure the binding affinity between the AXH domain and CIC in a quantitative manner, we assayed binding affinities using isothermal titration calorimetry (ITC). Wild-type $\mathrm{CIC}_{21}$ binds to the $\mathrm{AXH}$ domain with $\sim 100 \mathrm{nM} \mathrm{K}_{\mathrm{D}}$ affinity, while $\mathrm{CIC}_{21}$ mutants $\left(\mathrm{CIC}_{21 \_W 37 \mathrm{~A}}\right.$ and $\mathrm{CIC}_{21 \_L 40 S}$ show decreased affinity, with a $\mathrm{K}_{\mathrm{D}}$ in the micromolar range (Fig. 2D). We next measured the binding between wild-type $\mathrm{CIC}_{21}$ and the $\mathrm{AXH}$ mutants (Supplemental Fig. 4). Unexpectedly, the binding between $\mathrm{CIC}_{21}$ and the $\mathrm{AXH}_{\mathrm{V} 591 \mathrm{~A}} \mathrm{~S} 602 \mathrm{D}$ mutant shows endothermic kinetics. Moreover, the double mutants (AXH ${ }_{\text {V591A_L686E }}$ and $\mathrm{AXH}_{\text {S602D_L686E }}$, which fail to interact with $\mathrm{CIC}_{21}$ by MBP pull-down assay, show apparent binding affinities comparable with $\mathrm{AXH}_{\mathrm{WT}}$ to $\mathrm{CIC}_{21}$. We also measured the binding between wild-type $\mathrm{CIC}_{21}$ and $\mathrm{AXH}$ single mutants $\left(\mathrm{AXH}_{\mathrm{V} 591 \mathrm{~A}}, \mathrm{AXH}_{\mathrm{S602D}}\right.$, and $\left.\mathrm{AXH}_{\mathrm{L} 686 \mathrm{E}}\right)$ as well as a triple mutant (AXH V591A_S602D_L686E) (Supplemental Fig. 5). All single mutants show binding affinities comparable with the wild-type, and the triple mutant shows almost no binding. Furthermore, to investigate the dimerization status of the double mutants, we performed size exclusion chromatography and found that all double mutants elute as monomer forms, indicating that the dimerization interface was disrupted (Supplemental Fig. 6). The double mutants affect not only the homodimerization of the AXH domain, but also the hydrophobic interface between the AXH domain and CIC (Fig. 2A). This makes it difficult to interpret the binding between the $\mathrm{AXH}$ domain and $\mathrm{CIC}_{21}$ by ITC.

Therefore, we decided to directly measure the binding between the AXH mutants $\left(\mathrm{AXH}_{\mathrm{V} 591 \mathrm{~A} \_ \text {S602D, }}\right.$ $\mathrm{AXH}_{\mathrm{V} 591 \mathrm{~A} \_\mathrm{L} 686 \mathrm{E}}$, and $\mathrm{AXH}_{\mathrm{S602D}}$ L686E) and $\mathrm{CIC}_{21}$ using surface plasmon resonance (SPR), which measures the binding events only between the AXH domain and CIC. We immobilized biotinylated $\mathrm{CIC}_{21}$ peptide on a SA chip as a ligand and examined the bindings of the AXH domains as analytes. Consistent with the MBP pull-down assay, SPR analysis shows that the wild-type AXH domain binds to $\mathrm{CIC}_{21}$ with $\sim 40 \mathrm{nM} \mathrm{K}_{\mathrm{D}}$ affinity, while the mutant $\mathrm{AXH}$ variants are unable to bind to CIC (Fig. 2A [bottom panel], E). These data are consistent with the notion that CIC binds the hydrophobic pocket of the AXH domain and disrupts the homodimerization of the $\mathrm{AXH}$ domain.

\section{Reconfiguration of complex formation by ATXN1 and CIC}

Upon further examination of the structure, we noticed that there are three molecules of the $\mathrm{AXH}-\mathrm{CIC}_{21} \mathrm{com}-$ plexes in an asymmetric unit in the crystal. Among them, the structure of two $\mathrm{AXH}-\mathrm{CIC}_{21}$ complexes suggests a possible new form of ATXN1 dimerization mediated 
Kim et al.

A
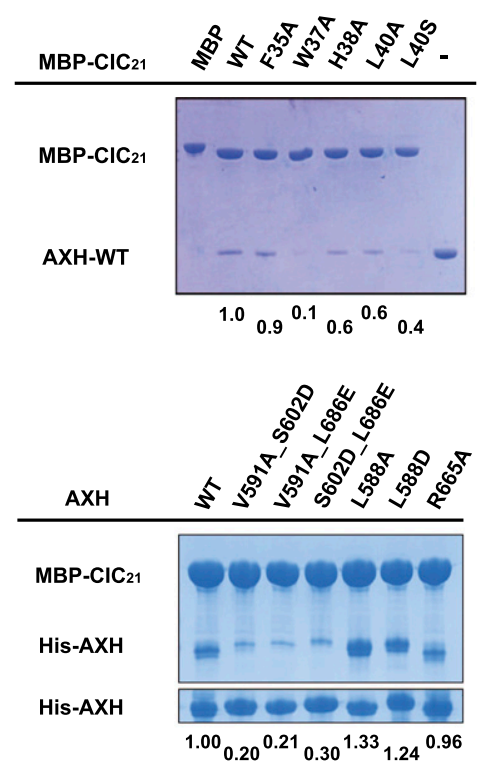

B

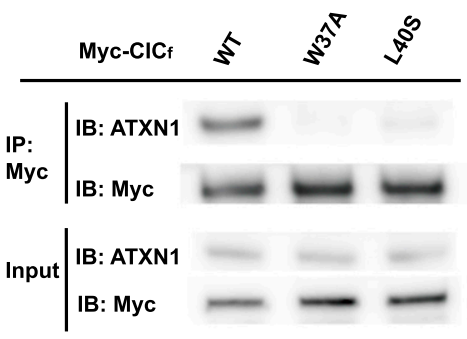

C

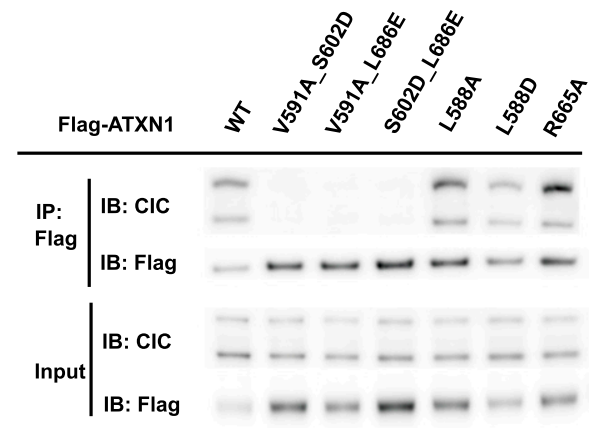

D

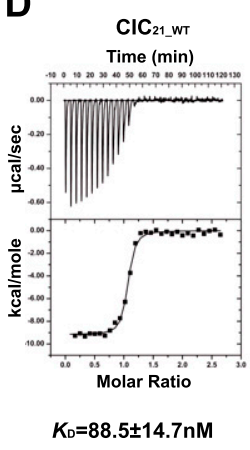

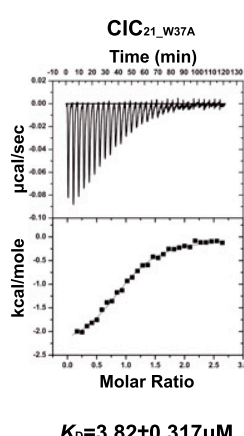

$K_{\mathrm{D}}=3.82 \pm 0.317 \mu \mathrm{M}$

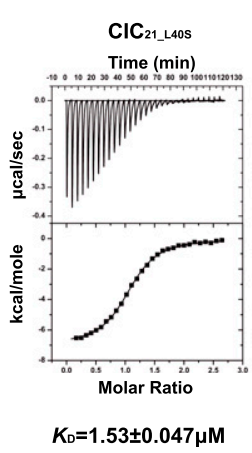

E

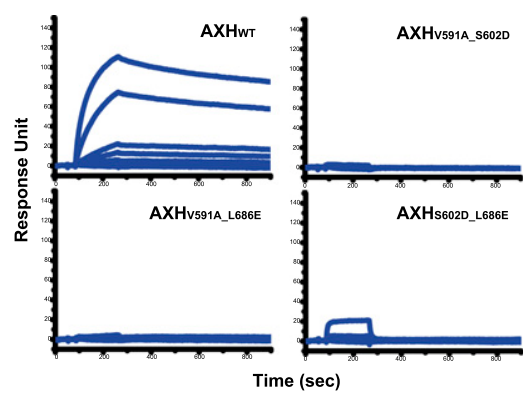

Figure 2. Mutation in critical residues disrupts the interaction between the ATXN1 and CIC both in vitro and in vivo. $(A$, top) MBP pull-

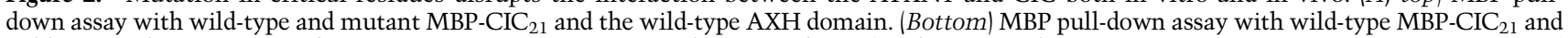
wild-type and mutant AXH domains. Mutations in MBP-CIC 21 (W37A and L40S) and His-AXH (V591A_S602D, V591A_L686E, or S602D_L686E) greatly disrupt the interaction. The same amounts of the AXH domains used for MBP pull-down were shown by His pull-down for His-AXH domains. The amount of bound AXH domain is quantified at the bottom of the gels. (B) In vivo binding between endogenous ATXN1 and Myctagged full-length $\mathrm{CIC}\left(\mathrm{CIC}_{\mathrm{f}}\right)$. One-hundred nanograms of each $\mathrm{CIC}_{\mathrm{f}}$ construct was used for transfection. $(C)$ In vivo binding between endogenous CIC and full-length wild-type and mutant ATXN1 (R566A is a negative control). The upper band of CIC is the longer isoform (CIC-L), and the lower band is the short isoform (CIC-S). Fifty nanograms of each Flag-ATXN1 construct was used for transfection. (D) ITC measurements between the $\mathrm{AXH}$ domain and $\mathrm{CIC}_{21} \mathrm{wT}_{1} \mathrm{CIC}_{21}$ w37A, and $\mathrm{AXH}-\mathrm{CIC}_{21} \mathrm{~L}_{40 \mathrm{~s}}$. $\mathrm{CIC}_{21}$ w37A and $\mathrm{AXH}-\mathrm{CIC}_{21}$ L40s have greatly reduced affinity to the AXH domain compared with $\mathrm{CIC}_{21}{ }_{-}$WT. $(E)$ SPR measurements of the binding affinities between $\mathrm{CIC}_{21}$ and the AXH domain. The $\mathrm{K}_{\mathrm{D}}$ for the binding between $\mathrm{CIC}_{21}$ and $\mathrm{AXH}_{\mathrm{WT}}$ is $\sim 40 \mathrm{nM}$. $\mathrm{AXH}_{\mathrm{V} 591 \mathrm{~A} \_ \text {S602D, }} \mathrm{AXH}_{\text {V591A_L686E, }}$ and $\mathrm{AXH}_{\text {S602D_L686E }}$ fail to bind to CIC 21 .

by the CIC dimer (Supplemental Fig. 7). To assess the biological relevance of this new form of AXH-CIC complex, we determined the crystal structure of the AXH domain bound to a longer CIC peptide (21-48 amino acids, $\mathrm{CIC}_{28}$ :MFVWTNVEPRSVAVFPWHSLVPFLAPSQ) at $3.15 \AA$ resolution by molecular replacement method using the $\mathrm{AXH}$ domain alone without $\mathrm{CIC}_{21}$ as a search model (Fig. 3; Supplemental Table 1). There are two $\mathrm{AXH}-\mathrm{CIC}_{28}$ molecules in an asymmetric unit of the crystals, which belong to a different space group with the $\mathrm{AXH}-\mathrm{CIC}_{21}$ crystals (Supplemental Table 1), indicating that the $\mathrm{AXH}-\mathrm{CIC}_{28}$ molecules have a different packing environment with the $\mathrm{AXH}-\mathrm{CIC}_{21}$. Indeed, the AXH domains make a new form of dimerization mediated by the $\mathrm{CIC}_{28}$ dimer (Fig. 3A). Two $\mathrm{CIC}_{28}$ molecules positioned in an anti-parallel manner make an intertwining interaction between each other, and these two inter- twined $\mathrm{CIC}_{28}$ molecules bridge two $\mathrm{AXH}$ domains. Specifically, hydrophobic residues (MFVW, 21-24 amino acids) of $\mathrm{CIC}_{28}$ interact with a hydrophobic patch composed of F632, V634, V645, and Y649 from the AXH domain as well as with $\mathrm{F} 43$ from the other $\mathrm{CIC}_{28}$ (Fig. 3B). Furthermore, four molecules (two $\mathrm{CIC}_{28}$ molecules and two AXH domains) interact together, forming a hydrophobic core composed of I580 and L588 from both AXH domains and V32 and V34 from both $\mathrm{CIC}_{28}$ molecules (Fig. 3C). To examine whether the AXH domain dimerization mediated by CIC occurs in a more physiological condition, we analyzed complex formation of $\mathrm{AXH}$ and CIC comparing the sizes of several AXH-CIC complexes by size exclusion chromatography (Fig. 4A). The wildtype $\mathrm{CIC}_{28}-\mathrm{AXH}$ domain elutes earlier than the $\mathrm{AXH}$ domain alone (homodimer), indicating that the AXH$\mathrm{CIC}_{28}$ complex forms a larger complex or a complex with 
A
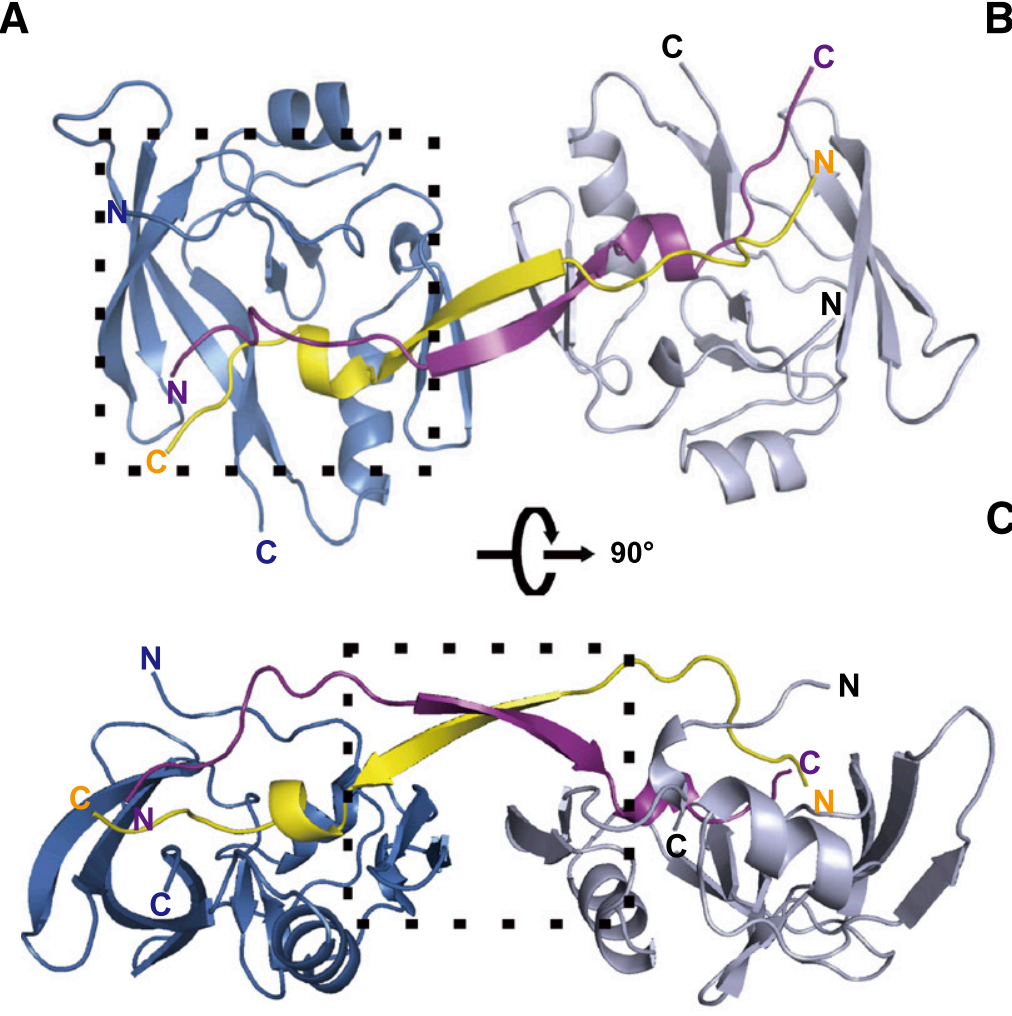

B
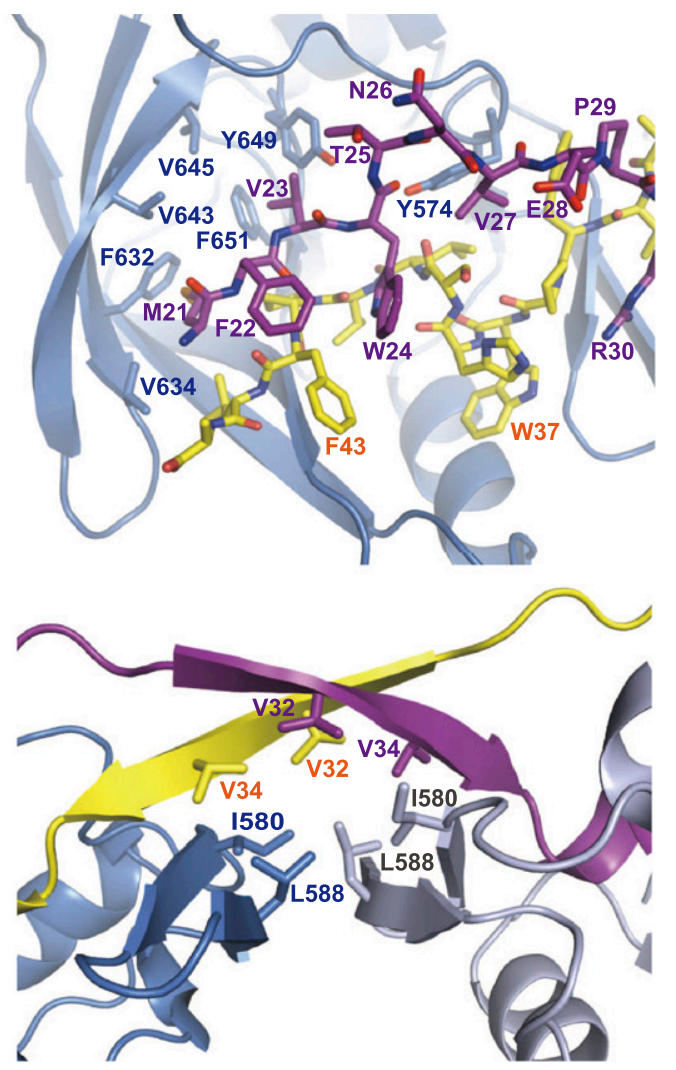

Figure 3. Crystal structure showing $\mathrm{CIC}_{28}$-mediated heterotetramer formation with the AXH domain. (A) Ribbon representation of the AXH$\mathrm{CIC}_{28}$ structure showing that $\mathrm{CIC}_{28}$ reconfigures the complex formation of the $\mathrm{AXH}$ domain (the $\mathrm{AXH}$ domain is shown in blue and light blue, and the $\mathrm{CIC}_{28}$ peptide is shown in yellow and purple). $(B)$ Detailed structure between $\mathrm{AXH}-\mathrm{CIC}_{28}$ and the other $\mathrm{CIC}_{28}$ peptide (shown in the dashed box in the top panel of $A)$. $(C)$ Detailed structure of the hydrophobic core where two AXH domains and two $\mathrm{CIC}_{28}$ peptides interact (shown in the dashed box in the bottom panel of $A$ ).

extended conformation. Consistent with this observation, the $\mathrm{CIC}_{28}$-mediated $\mathrm{AXH}$ dimer structure has an extended shape compared with the AXH homodimer. We then generated a triple mutation in $\mathrm{CIC} / \mathrm{CIC}_{28 \_\mathrm{AEA}}$ : F22A_V23E_W24A), which would affect the CIC-mediated $\mathrm{AXH}$ dimerization but not AXH-CIC interaction. We also generated AXH mutants (L588A and L588D) to disrupt the hydrophobic core where four molecules junction without affecting the CIC-binding pocket at the AXH domain (Fig. 2A,C). All of the mutants elute at similar fractions where $\mathrm{AXH}-\mathrm{CIC}_{21}$ elutes, which is shown to be a monomer, suggesting that the mutations disrupt the CIC-mediated AXH dimerization, resulting in a AXH-CIC monomer. To analyze the complex formation more quantitatively, we measured the size of the complexes using size exclusion chromatography multi-
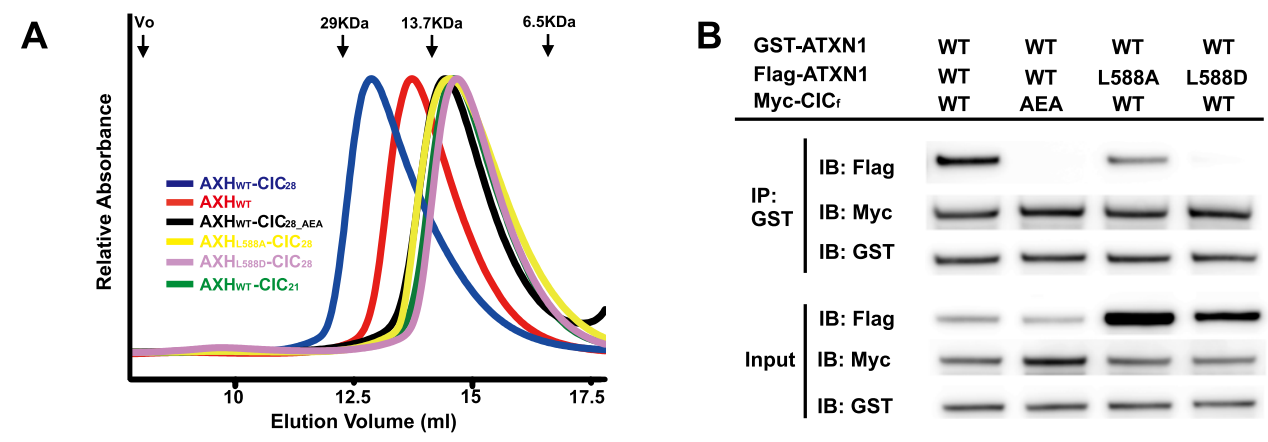

Figure 4. CIC mediates the dimerization of ATXN1 to form heterotetramers both in vitro and in vivo. (A) Size exclusion chromatography analysis of AXH-CIC complexes. The AXH domain alone forms a dimer. AXH-CIC 28 elutes earlier than the AXH domain alone, suggesting that AXH-CIC 28 forms a larger complex. (Blue) AXH-CIC 28 (red) the AXH domain alone; (black) AXH-CIC 28 AEA; (yellow) AXH L 588 A-CIC $_{28 ;}$ (pink) $\mathrm{AXH}_{\mathrm{L} 588 \mathrm{D}}-\mathrm{CIC}_{28}$; (green) $\mathrm{AXH}-\mathrm{CIC}_{21}$. The black arrows indicate size markers and void volume $\left(\mathrm{V}_{\mathrm{o}}\right)$. $(B)$ In vivo binding showing that $\mathrm{CIC}$ mediates ATXN1 dimerization. Wild-type GST-ATXN1 (100 ng), wild-type and mutant Flag-ATXN1 (80 ng), and wild-type and mutant CIC $\mathrm{f}_{\mathrm{f}}(1 \mu \mathrm{g})$ were transfected. GST-ATXN1 can pull down Flag-ATXN1 in the presence of wild-type CIC $_{\mathrm{f}}$ and wild-type Flag-ATXN1, but the interaction is abolished or reduced with mutant $\mathrm{CIC}_{\mathrm{f}}$ or mutant Flag-ATXN1. 
angle light scattering (SEC-MALS) (Supplemental Fig. 8). SEC-MALS analysis shows that the molecular weight of the $\mathrm{AXH}_{\mathrm{WT}}-\mathrm{CIC}_{28}$ shows $33.8 \mathrm{kDa}$, indicating that $\mathrm{AXH}_{\mathrm{WT}}-\mathrm{CIC}_{28}$ forms a heterotetramer of $\mathrm{AXH}_{\mathrm{WT}}-\mathrm{CIC}_{28}$, whereas $\mathrm{AXH}_{\mathrm{L} 588 \mathrm{D}}-\mathrm{CIC}_{28}$ shows a heterodimer size. Interestingly, $\mathrm{AXH}_{\mathrm{WT}}-\mathrm{CIC}_{28}$ AEA and $\mathrm{AXH}_{\mathrm{L} 588 \mathrm{~A}}-\mathrm{CIC}_{28}$ show slightly higher molecular weights than their heterodimer sizes, suggesting the existence of partial heterotetramers. Collectively, SEC-MALS data with the gel filtration analysis indicate that the CIC dimer can reconfigure the ATXN1 dimer and form a heterotetramer with ATXN1s.

To determine whether the structural data and in vitro studies regarding the $\mathrm{CIC}_{28}$-mediated complex formation of $\mathrm{AXH}$ reflect the in vivo complex formation by fulllength ATXN1 and CIC, we transfected GST-ATXN1 and Flag-ATXN1 with saturated amounts of wild-type Myc$\mathrm{CIC}_{\mathrm{f}}\left(\mathrm{CIC}_{\mathrm{f}_{-} \mathrm{WT}}\right)$ or Myc-CIC mutant $\left(\mathrm{CIC}_{\mathrm{f}} \mathrm{AEA}\right.$ : F22A V23E_W24A) and examined CIC-mediated ATXN1 dimerization (Fig. 4B). Figure 4B shows that wild-type GSTATXN1 is able to bring down Flag-ATXN1 when cotransfected with $\mathrm{CIC}_{\mathrm{f}_{-} \text {WT }}$ but not with mutant $\mathrm{CIC}_{\mathrm{f}_{\_} \mathrm{AEA}}$ suggesting that the dimer formation between GSTATXN1 and Flag-ATXN1 is mediated by CIC and that the AEA mutation in CIC disrupts ATXN1 dimerization. These data are consistent with the structural observation that the hydrophobic residues (F22, V23, and W24) are critical for CIC-mediated dimerization of ATXN1. Next, we transfected wild-type GST-ATXN1 and Flag-ATXN1 mutants (L588A and L588D) with CIC $_{\mathrm{f}_{-} \mathrm{WT}}$ and examined the CIC-mediated dimerization of ATXN1. The mutants (L588A and L588D) would affect the AXH dimerization mediated by CIC but not the interaction between $\mathrm{AXH}$ and CIC (Fig. 2C). Figure 4B shows that wild-type GSTATXN1 failed to pull down the Flag-ATXN1 mutant

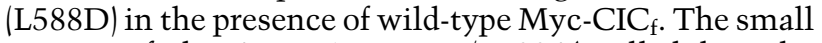
amount of Flag-ATXN1 mutant (L588A) pulled down by GST-ATXN1 was likely due to the hydrophobic property of alanine substitution. Collectively, these data show that the CIC binding to ATXN1 disrupts the homodimerization and reconfigures to make a CIC-mediated ATXN1 dimer complex.

The interaction between polyQ-expanded ATXN1 and CIC plays a critical role in SCA1 pathogenesis (Fryer et al. 2011). The notion that the binding of CIC to ATXN1 substantially reconfigures the arrangement of ATXN1 prompted us to propose that the rearrangement of ATXN1 homodimer to the CIC-mediated ATXN1 complex might affect the interaction with one of the many other binding partners (Lim et al. 2008). Previous work showed that the level of CIC protein is reduced in the absence of ATXN1, and the level of ATXN1 is also reduced upon reduction of CIC in a dose-dependent manner (Fryer et al. 2011).

The crystal structure in this study reveals that the ATXN1 dimer is mediated by the CIC dimer and that the CIC dimer is mediated by the ATXN1 dimer, indicating that the formation of the ATXN1-CIC complex is highly interdependent. These observations suggest that the protein stabilities of ATXN1 and CIC might be contingent on ATXN1-CIC complex formation. Recently, it was shown that the polyQ in Huntington's disease protein modulates the activity of its endogenous binding partner, Polycombrepressive complex2 (Seong et al. 2010). Furthermore, it was shown that in SCA1 knock-in mice, some CIC target genes are hyperrepressed, while other CIC target genes are derepressed (Fryer et al. 2011). Therefore, it is tantalizing to propose that the expanded polyQ in ATXN1CIC-CIC-ATXN1 configuration may alter the transcriptional repressor activity of CIC, which may be involved in SCA1 pathology. However, the exact mechanism of SCA1 pathology regarding the ATXN1 and CIC interaction needs to be further investigated.

This study provides the first structural and molecular insights into the interaction between one polyQ disease protein, ATXN1, and its endogenous binding partner, CIC. Our findings underscore the importance of domains beyond the glutamine tract for interactions that modulate disease pathogenesis. Given the in vivo benefits of reducing CIC levels in the SCA1 mouse model (Fryer et al. 2011), the identification of the surface mediating the ATXN1-CIC interaction opens up the possibility of developing agents that target the ATXN1-CIC interactions to modulate SCA1 pathogenesis.

\section{Materials and methods}

\section{Protein expression and purification}

The AXH domain (563-689 amino acids) from human ATXN1 was cloned into a pET28a vector, generating an $\mathrm{N}$-terminal His tag. The AXH domain was expressed in Escherichia coli BL21(DE3) and purified with an Niaffinity column. The $\mathrm{N}$-terminal His tag was cleaved by TEV protease (Life Technologies) and further purified with a Hitrap Q (GE Healthcare) anion exchange chromatography and with a Superdex 75 (GE Healthcare) size exclusion chromatography. The AXH mutants and the N-terminaldeleted AXH were purified as the wild-type AXH domain. For the Sesubstituted AXH domain, we decided to mutate Ile580 and Ile605 to methionines to obtain better phase information using a QuikChange sitedirected mutagenesis kit (Stratagene), and the Se-Substituted AXH domain was purified in the same way as the wild-type.

\section{Crystallization and structure determination}

The AXH-CIC ${ }_{21}$ complex was crystallized in the presence of $3 \mathrm{mM} \mathrm{CIC}_{21}$ peptide (EPRSVAVFPWHSLVPFLAPSQ) in a reservoir solution containing $0.1 \mathrm{M}$ calcium chloride, $24 \%(\mathrm{v} / \mathrm{v})$ PEG 3350 , and $4 \%(\mathrm{v} / \mathrm{v})$ pentaerythritol ethoxylate by the hanging drop vapor diffusion method at $20^{\circ} \mathrm{C}$. The $\mathrm{AXH}-\mathrm{CIC}_{28}$ complex was crystallized in the presence of $\mathrm{CIC}_{28}$ peptide (MFVWTNVEPRSVAVFPWHSLVPFLAPSQ) in a mother liquor containing $1.6 \mathrm{M} \mathrm{NaCl}, 3 \%$ (v/v) glycerol, $16 \%$ (w/v) PEG 3350, and $1 \mathrm{mM}$ L-glutathione. $\mathrm{AXH}-\mathrm{CIC}_{21}$ crystals were soaked in a cryo-solution containing 26\% (w/v) PEG3350 and 10\% (v/v) glycerol. For AXH-CIC 28 , the mother liquor solution was used as a cryo-protectant. X-ray diffraction data were collected at the beamline 17A at Photon Factory and the beamline $5 \mathrm{C}$ at the Pohang Accelerator Laboratory. The data were processed using HKL2000. The AXH-CIC 21 structure was determined by Se-SAD method using Phenix and was refined using the CNS program. The AXH-CIC 28 structure was solved by the molecular replacement method using the AXH apo-structure as a search model and refined using the CNS program. The structure factors and coordinates of the structures were deposited at the Protein Data Bank (http://www.rcsb.org; PDB ID 4J2J for AXH-CIC 21 and 4J2L for $\mathrm{AXH}-\mathrm{CIC}_{28}$ ).

\section{Pull-down assay}

MBP-tagged wild-type and mutant CIC21 were expressed in E. coli BL21(DE3) and purified using amylose (New England Biolabs) resin. The purified wild-type $\mathrm{AXH}$ domain was incubated with each MBP-tagged $\mathrm{CIC}_{21}$ bound to amylose resin. The amylose resin was washed using the lysis buffer. The interaction was analyzed by SDS-PAGE. For wild-type $\mathrm{MBP}-\mathrm{CIC}_{21}$ and the mutant $\mathrm{AXH}$ binding, His-tagged wild-type or mutant $\mathrm{AXH}$ domains and $\mathrm{MBP}$-tagged $\mathrm{CIC}_{21}$ were coexpressed in E. coli BL21(DE3) cells and purified using amylose (New England Biolabs) resin or 
Ni-NTA agarose (Qiagen) resin. Using SDS-PAGE, the interaction was analyzed. The pull-downs were repeated three times with separately grown cells. The amount of proteins was quantified using ChemiDoc MP (Bio-Rad) using Image Lab software.

\section{ITC}

The interaction of $\mathrm{AXH}$ and $\mathrm{CIC}_{21}$ was measured using ITC using VP-ITC (MicroCal) at $25^{\circ} \mathrm{C}$. The $\mathrm{AXH}$ domain and $\mathrm{CIC}_{21}$ peptide were prepared in a buffer containing $100 \mathrm{mM} \mathrm{NaCl}$ and $20 \mathrm{mM}$ HEPES (pH 7.5). The wildtype or mutant $\mathrm{AXH}$ domains were added at $25 \mu \mathrm{M}$ concentration in the sample chamber and titrated with $\mathrm{CIC}_{21}$ peptide with $500 \mu \mathrm{M}$ concentration. Data were analyzed using the Origin program.

\section{$S P R$}

The interactions between the $\mathrm{AXH}$ domain and $\mathrm{CIC}_{21}$ were measured by SPR using Biacore 3000 (GE Healthcare). Biotinylated $\mathrm{CIC}_{21}$ was immobilized as a ligand on a Sensor Chip SA with 80RU, and the immobilized surface was stabilized with a regeneration buffer containing $50 \mathrm{mM} \mathrm{NaOH}$. The wild-type or mutant $\mathrm{AXH}$ domains were used as analytes $(0,5,10,25,50$, 200 , and $400 \mathrm{nM}$ ) using a buffer containing $300 \mathrm{mM} \mathrm{NaCl}, 20 \mathrm{mM}$ HEPES (pH 7.5), and 1mM EDTA as a running buffer. The association was measured for $3 \mathrm{~min}$, and the dissociation was measured for $12 \mathrm{~min}$. The surface of chip was regenerated with two injections of the regeneration buffer for 4 min each time. The data were analyzed using BiaEvaluation software (GE Healthcare).

\section{Dimerization assay}

The size of AXH-CIC complexes were analyzed with a Superdex 75 10/300 GL (GE Healthcare) size exclusion chromatography equilibrated with a buffer containing $100 \mathrm{mM} \mathrm{NaCl}$ and $20 \mathrm{mM}$ HEPES (pH 7.5).

\section{In vivo binding assay}

Mammalian expression vectors Flag-ATXN1 (Flag-Atxn1[2Q]), CIC $_{\mathrm{f}_{-} \text {WT }}$ (Myc-CicS), and GST-ATXN1 (GST-Atxn1[2Q]) have been described previously (Chen et al. 2003; Lim et al. 2008). $\mathrm{CIC}_{\mathrm{f}_{\mathrm{L}} \mathrm{wT}}\left(\mathrm{Myc}-\mathrm{CIC}_{\mathrm{f}}\right.$ ) was generated by PCR cloning of mouse Cic cDNA (DNASU, MmCD00083297) into pCMV-Myc vector (Clontech). The $\mathrm{CIC}_{\mathrm{f}_{-} \mathrm{AEA}}$ construct was modified from $\mathrm{CIC}_{\mathrm{f}} \mathrm{wT}$ by PCR cloning using primers with a mutagenized sequence. The other constructs were made by site-directed mutagenesis with QuickChange II XL site-directed mutagenesis kit (Agilent) using either Flag-ATXN1 or $\mathrm{CIC}_{\mathrm{f}_{-} \text {wT }}$ as a template. HEK293Ta cells were cultured in DMEM medium with $10 \%$ fetal bovine serum and were plated in six-well plates $1 \mathrm{~d}$ before transfection. On the day of transfection, corresponding DNA constructs were transfected with Lipofectamine 2000 (Invitrogen). Two days later, cells were lysed for $20 \mathrm{~min}$ at $4^{\circ} \mathrm{C}$ in lysis buffer $(150 \mathrm{mM}$ $\mathrm{NaCl}, 50 \mathrm{mM}$ Tris-HCl at $\mathrm{pH} 7.5,1 \mathrm{mM}$ EDTA, $0.1 \%$ Triton X-100, 0.1\% Tween-20, complete protease inhibitor cocktail [Roche], PhosSTOP phosphatase inhibitor cocktail [Roche]). After centrifugation, $500 \mu \mathrm{g}$ of soluble proteins was used for immunoprecipitation with glutathione Sepharose $4 \mathrm{~B}$ beads (GE), Anti-Flag M2 affinity gel (Sigma), or mouse monoclonal antic-Myc antibody (Sigma, clone 9E10) and protein G Sepharose 4 Fast Flow beads (GE). The pellets were resuspended in $2 \times$ sample buffer after being washed three times in washing buffer $(150 \mathrm{mM} \mathrm{NaCl}, 50 \mathrm{mM}$ Tris-HCl at pH 7.5, 1 mM EDTA, complete protease inhibitor cocktail [Roche], PhosSTOP phosphatase inhibitor cocktail [Roche]). The protein samples were loaded on NuPage $4 \%-12 \%$ Bis-Tris gel (Invitrogen) and transferred to Trans-Blot nitrocellulose membrane (Bio-Rad). Guinea pig polyclonal antiCIC (126) (Lam et al. 2006), rabbit polyclonal anti-ATXN1 (11750) (Servadio et al. 1995), mouse monoclonal anti-Flag M2 (Sigma), rabbit polyclonal antiGST (Sigma), and mouse monoclonal anti-c-Myc (Sigma, clone 9E10) were used as primary antibodies.

\section{Acknowledgments}

We thank the members of the Song laboratory for discussion. We also thank J. Park, D. Kim, H.-C. Shin, and B. Ku for technical assistant; S.-B. Hong and H.K. Song for MALS analysis; and H.-Y. Kim at Korea Basic Science Institute for initial crystal screening. The X-ray data were collected at Photon Factory and Pohang Accelerator Laboratory. This work was partially supported by the World Class University (WCU) program grant R31-2008-000-10071-0, and grants 2011-0031416, 20110020334, and 2011-0031955 to J.S. E.K. and J.S. conceived of the study. E.K, H.-C.L., H.Y.Z, and J.S. designed the experiments. E.K. and J.S. performed structural studies and in vitro binding experiments. H.-C.L. performed in vivo binding experiments. E.K., H.-C.L., H.Y.Z., and J.S. analyzed the data, and E.K. and J.S. wrote the paper. H.Y.Z. is an investigator with the Howard Hughes Medical Institute, and portions of the work were supported by NIH grant NS27699 to H.Y.Z.

\section{References}

Burright EN, Davidson JD, Duvick LA, Koshy B, Zoghbi HY, Orr HT. 1997. Identification of a self-association region within the SCA1 gene product, ataxin-1. Hum Mol Genet 6: 513-518.

Chen HK, Fernandez-Funez P, Acevedo SF, Lam YC, Kaytor MD, Fernandez MH, Aitken A, Skoulakis EM, Orr HT, Botas J, et al. 2003. Interaction of Akt-phosphorylated ataxin-1 with 14-3-3 mediates neurodegeneration in spinocerebellar ataxia type 1. Cell 113: 457-468.

Chen YW, Allen MD, Veprintsev DB, Lowe J, Bycroft M. 2004. The structure of the AXH domain of spinocerebellar ataxin-1. J Biol Chem 279: $3758-3765$.

Fryer JD, Yu P, Kang H, Mandel-Brehm C, Carter AN, Crespo-Barreto I, Gao Y, Flora A, Shaw C, Orr HT, et al. 2011. Exercise and genetic rescue of SCA1 via the transcriptional repressor Capicua. Science 334: 690-693.

Lam YC, Bowman AB, Jafar-Nejad P, Lim J, Richman R, Fryer JD, Hyun ED, Duvick LA, Orr HT, Botas J, et al. 2006. ATAXIN-1 interacts with the repressor Capicua in its native complex to cause SCAl neuropathology. Cell 127: 1335-1347.

Lim J, Crespo-Barreto J, Jafar-Nejad P, Bowman AB, Richman R, Hill DE, Orr HT, Zoghbi HY. 2008. Opposing effects of polyglutamine expansion on native protein complexes contribute to SCA1. Nature 452: 713-718.

Orr HT, Chung MY, Banfi S, Kwiatkowski TJ Jr, Servadio A, Beaudet AL, McCall AE, Duvick LA, Ranum LP, Zoghbi HY. 1993. Expansion of an unstable trinucleotide CAG repeat in spinocerebellar ataxia type 1 . Nat Genet 4: 221-226.

Seong IS, Woda JM, Song JJ, Lloret A, Abeyrathne PD, Woo CJ, Gregory G, Lee JM, Wheeler VC, Walz T, et al. 2010. Huntingtin facilitates polycomb repressive complex 2. Hum Mol Genet 19: 573-583.

Servadio A, Koshy B, Armstrong D, Antalffy B, Orr HT, Zoghbi HY. 1995. Expression analysis of the ataxin-1 protein in tissues from normal and spinocerebellar ataxia type 1 individuals. Nat Genet 10: 94-98.

Tsuda H, Jafar-Nejad H, Patel AJ, Sun Y, Chen HK, Rose MF, Venken KJ, Botas J, Orr HT, Bellen HJ, et al. 2005. The AXH domain of Ataxin-1 mediates neurodegeneration through its interaction with Gfi-1/Senseless proteins. Cell 122: 633-644. 


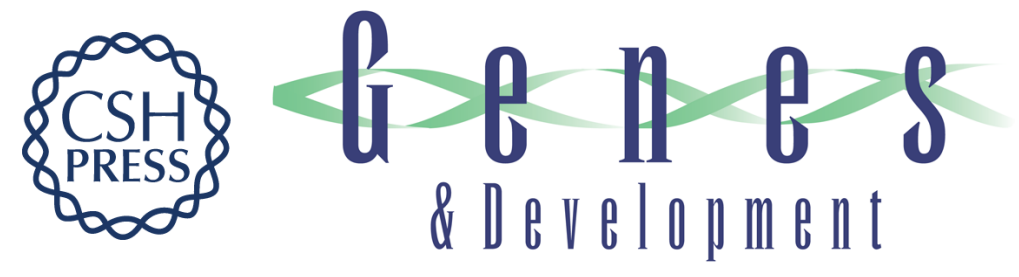

\section{Structural basis of protein complex formation and reconfiguration by polyglutamine disease protein Ataxin-1 and Capicua}

Eunji Kim, Hsiang-Chih Lu, Huda Y. Zoghbi, et al.

Genes Dev. 2013, 27:

Access the most recent version at doi:10.1101/gad.212068.112

Supplemental
Material $\quad$ http://genesdev.cshlp.org/content/suppl/2013/03/19/27.6.590.DC1

References This article cites 10 articles, 2 of which can be accessed free at:

http://genesdev.cshlp.org/content/27/6/590.full.html\#ref-list-1

License

Email Alerting Receive free email alerts when new articles cite this article - sign up in the box at the top

Service

right corner of the article or click here.

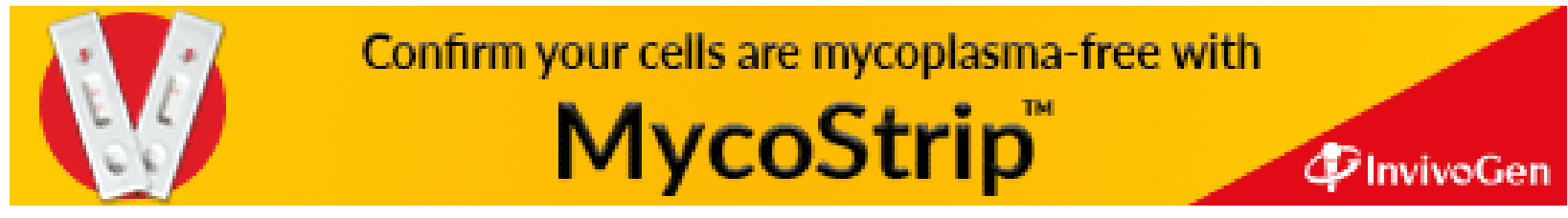

